\title{
Application of Isotropic Finite Strain Theory to Ultrasonic and Seismological Data ${ }^{1}$
}

\author{
Charles Sammis, Don Anderson, and Tom Jordan \\ Seismological Laboratory, California Institute of Technology, Pasadena 91109
}

The finite strain expressions, originally derived by F. Birch (1938), for the compressional and shear velocities in an isotropic solid under hydrostatic pressure, when rederived to be complete to first order in the strain, are functions of both the second-and third-order elastic constants. The extra terms appearing in the complete first-order theory for the velocities and their pressure derivatives are of the order of the ordinary elastic constants. There is no longer any discrepancy between first-order finite strain theory and ultrasonic and seismic data.

In a recent article, Birch [1969] questioned the ultrasonically measured velocity derivatives of spinel, garnet, and forsterite, since they were in poor agreement with the expressions

$$
\begin{aligned}
& \left(\frac{1}{V_{P}} \partial V_{P}\right)_{0}=\frac{1}{6 K_{0}} \frac{13 \lambda_{0}+14 \mu_{0}}{\lambda_{0}+2 \mu_{0}} \\
& \left(\frac{1}{V_{S}} \frac{\partial V_{S}}{\partial P}\right)_{0} \\
& \frac{1}{6 K_{0}} \frac{3 \lambda_{0}+6 \mu_{0}}{\mu_{0}}
\end{aligned}
$$

These equations were derived by Birch [1938] using Murnaghan's theory of finite strain. Part of the reason for the poor agreement with the ultrasonic experiments is that these expressions are not complete. The complete expressions to first order in the strain are

$$
\begin{aligned}
& \left(\frac{1}{V_{P}} \frac{\partial V_{P}}{\partial P}\right)_{0} \\
& \quad=\frac{1}{6 K_{0}} \frac{13 \lambda_{0}+14 \mu_{0}-18 l-4 m}{\lambda_{0}+2 \mu_{0}} \\
& \left(\frac{1}{V_{S}} \frac{\partial V_{S}}{\partial P}\right)_{0} \\
& \quad=\frac{1}{6 K_{0}} \frac{3 \lambda_{0}+6 \mu_{0}+\frac{3}{2} m+\frac{1}{2} n}{\mu_{0}}
\end{aligned}
$$

The constants $l, m$, and $n$ are the third-order coefficients in the expansion of the elastic energy density in powers of the strain invariants

\footnotetext{
${ }^{1}$ Contribution 1724, California Institute of Technology, Pasadena.
}

Copyright (c) 1970 by the American Geophysical Union.

$$
\begin{array}{r}
\rho_{0} \phi=\frac{\lambda_{0}+2 \mu_{0}}{2} I_{1}{ }^{2}-2 \mu_{0} I_{2}+l I_{1}{ }^{3} \\
+m I_{1} I_{2}+n I_{3}+O\left(\epsilon^{4}\right)
\end{array}
$$

The three invariants of the Eulerian strain tensor are given by

$$
\begin{aligned}
& I_{1}=\epsilon_{i i} \\
& I_{2}=\frac{1}{2}\left(\epsilon_{i i} \epsilon_{i j}-\epsilon_{i j} \epsilon_{i i}\right) \\
& I_{3}=\frac{1}{6}\left(\epsilon_{i i} \epsilon_{i j} \epsilon_{k k_{i}}-3 \epsilon_{i j} \epsilon_{i j} \epsilon_{k k}+2 \epsilon_{i j} \epsilon_{i k} \epsilon_{k i}\right) \\
& i \neq j \neq k
\end{aligned}
$$

The result that the pressure derivatives of the velocity are not completely determined by $\lambda_{0}$ and $\mu_{0}$ is intuitively reasonable. Stated another way, equations 1 and 2 imply that two materials that have the same $\lambda_{0}$ and $\mu_{0}$ would have the same logarithmic velocity derivative. The thirdorder isotropic constants $l, m$, and $n$ in equations 3 and 4 remove this unrealistic constraint.

The derivation of equations 3 and 4 is identical to Birch's [1938] derivation of (1) and (2) in every detail except one: the expansion of the strain energy density, equation 5 , is not truncated after the second-order terms, but is retained to third order in $\epsilon$ as written.

Following Birch, the compressional and shear velocities in an isotropic material subjected to a finite hydrostatic strain are

$$
\begin{aligned}
& V_{P}{ }^{2}=A(1+\alpha)^{2} / \rho_{0} \\
& V_{S}{ }^{2}=C(1+\alpha)^{2} / 2 \rho_{0}
\end{aligned}
$$

where 


$$
\begin{aligned}
A=\rho[ & {\left[( 1 - 2 \epsilon ) \left(\frac{\partial^{2} \phi}{\partial I_{1}^{2}}+4 \epsilon \frac{\partial^{2} \phi}{\partial I_{1} \partial I_{2}}\right.\right.} \\
& +2 \epsilon^{2} \frac{\partial^{2} \phi}{\partial I_{1}} \frac{\partial I_{3}}{\partial I^{2} \frac{\partial^{2} \phi}{\partial I_{2}^{2}}} \\
& \left.+4 \epsilon^{3} \frac{\partial^{2} \phi}{\partial I_{2} \partial I_{3}}+\epsilon^{4} \frac{\partial^{2} \phi}{\partial I_{3}^{2}}\right) \\
& \left.-3\left(\frac{\partial \phi}{\partial I_{1}}+2 \epsilon \frac{\partial \phi}{\partial I_{2}}+\epsilon^{2} \frac{\partial \phi}{\partial I_{2}}\right)\right] \\
C=\rho & {\left[-2 \frac{\partial \phi}{\partial I_{1}}-(1+2 \epsilon) \frac{\partial \phi}{\partial I_{2}}-\epsilon \frac{\partial \phi}{\partial I_{3}}\right] }
\end{aligned}
$$

Here $\epsilon$ is the finite hydrostatic strain $(\epsilon=-\alpha$ $-\alpha^{2} / 2, \rho / \rho_{0}=(1-2 \epsilon)^{3 / 2}$ and $1 /(1+\alpha)$ is the factor by which each line in the crystal is bydrostatically shortened.

By taking the indicated partial derivatives of $\phi$ and arranging the terms in ascending powers of $\epsilon$, we get

$$
\begin{aligned}
& V_{P}{ }^{2}=(1-2 \epsilon)^{5 / 2} \\
& \cdot\left[\lambda_{0}+2 \mu_{0}-\epsilon\left(11 \lambda_{0}+10 \mu_{0}-18 l-4 m\right)\right. \\
& \left.-\epsilon^{2}(117 l+35 m+3 n)\right] / \rho_{0} \\
& V_{S}{ }^{2}=(1-2 \epsilon)^{5 / 2} \\
& \cdot\left[2 \mu_{0}-\epsilon\left(6 \lambda_{0}+8 \mu_{0}+3 m+n\right)\right. \\
& \left.-\epsilon^{2}(54 l+12 m)\right] / 2 \rho_{0}
\end{aligned}
$$

Because of the differentiation in the calculation of $A$ and $C$, the third-order coefficients $l$, $m$, and $n$ appear with $\lambda_{0}$ and $\mu_{0}$ to the first order in $\epsilon$. For the same reason, the coefficients of the $\epsilon^{2}$ terms are incomplete. The complete terms would contain fourth-order constants ignored in the truncation of equation 5 after the $\epsilon^{3}$ terms. By truncating the free energy expansion after the second-order terms, Birch got only the $\lambda_{0}$ and $\mu_{0}$ contribution to the $\epsilon$ terms. Hughes and Kelly [1953] derived equations analogous to (9) in Lagrangian coordinates having the same form; i.e., the third-order coefficients appear to first order in the Lagrangian hydrostatic strain $\eta$.

Upon computing the bulk modulus $K / \rho=$ $V_{P}{ }^{2}-(4 / 3) V_{S}{ }^{2}$ by using $(9)$, we obtain

$$
\begin{aligned}
& K=(1-2 \epsilon)^{5 / 2} \\
& \quad \cdot\left[K_{0}-\epsilon\left(7 K_{0}-18 l-6 m-\frac{2}{3} n\right)\right. \\
& \left.-\epsilon^{2}(81 l+27 m+3 n)\right]
\end{aligned}
$$

which is identical to the expression given by Birch [1952]:

$$
\begin{aligned}
& K=K_{0}(1+2 f)^{5 / 2} \\
& \cdot[1+7 f-2 \xi f(2-9 f)]
\end{aligned}
$$

where $f=-\epsilon$ and $\xi=[18 l+6 m+2 / 3 n] /$ $4 K_{0}$. Note that the $f^{2}$ term in (11) is incomplete, being composed of the incomplete $\epsilon^{2}$ terms in the velocities. The pressure derivatives given by (3) and (4) are computed from (9) and from the expression for pressure given by Birch [1952]:

$$
P=-3 K_{0} \epsilon(1-2 \epsilon)^{5 / 2}(1+2 \epsilon \xi)
$$

Given only a hydrostatic finite strain, it is not possible to determine $l, m$, and $n$ individually, but only the combinations

$$
\begin{aligned}
& \xi=18 l+4 m \\
& \eta=\frac{1}{2}(3 m+n)
\end{aligned}
$$

which appear in the velocity derivatives. Since

$$
(d K / d P)_{0}=4-\frac{4}{3} \xi
$$

and

$$
\xi=\frac{18 l+6 m+\frac{2}{3} n}{4 K_{0}}=\frac{\zeta+\frac{4}{3} \eta}{4 K_{0}}
$$

equation 14 is linearly dependent on equations 3 and 4 . For most geophysical purposes, however, $\zeta$ and $\eta$ are sufficient. The parameters are given in Table 1 for a number of solids.

The most serious objection to finite strain theory is that one is never certain as to the convergence of the expressions for the velocities (9) or the bulk modulus (11). The coeffcient of the $\epsilon$ term is typically an order of magnitude larger than the leading term, and the coefficient of the $\epsilon^{2}$ term, although incomplete, appears to be an order of magnitude larger still. Therefore, these expressions are probably insufficient for $\epsilon>0.1$, which is roughly the strain at the base of the mantle. For self-consistent analyses, the $\epsilon^{2}$ terms, being incomplete, should not be retained. The expressions should be used in the form

$$
\begin{aligned}
& V_{P}{ }^{2}=\left(1 / \rho_{0}\right)(1-2 \epsilon)^{5 / 2} \\
& \cdot\left[\lambda_{0}+2 \mu_{0}-\epsilon\left(11 \lambda_{0}+10 \mu_{0}-\zeta\right)\right] \\
& V_{S}{ }^{2}=\left(1 / \rho_{0}\right)(1-2 \epsilon)^{5 / 2} \\
& \cdot\left[\mu_{0}-\epsilon\left(3 \lambda_{0}+4 \mu_{0}+\eta\right)\right] \\
& K=(1-2 \epsilon)^{5 / 2} K_{0}[1-\epsilon(7-4 \xi)] \\
& P=-3 K_{0} \epsilon(1-2 \epsilon)^{5 / 2}(1+2 \epsilon \xi)
\end{aligned}
$$


TABLE 1. Ultrasonic Data for the Velocity Derivatives, Bulk Modulus, and Shear Modulus

\begin{tabular}{|c|c|c|c|c|c|c|}
\hline Compound & $\begin{array}{c}\left(\frac{1}{V_{P}}\right)\left(\frac{d V_{P}}{d P}\right) \\
10^{-12} \\
\mathrm{~cm}^{2} / \text { dyne }\end{array}$ & $\begin{array}{c}\left(\frac{1}{V_{S}}\right)\left(\frac{d V_{S}}{d P}\right) \\
10^{-12} \\
\mathrm{~cm}^{2} / \text { dyne }\end{array}$ & $\begin{array}{c}K_{\dot{s}} \\
10^{12} \\
\text { dynes } / \mathrm{cm}^{2}\end{array}$ & $\begin{array}{c}\mu, \\
10^{12} \\
\text { dynes } / \mathrm{cm}^{2}\end{array}$ & $\begin{array}{c}\zeta \\
10^{12} \\
\text { dynes } / \mathrm{cm}^{2}\end{array}$ & $\begin{array}{c}\eta_{1} \\
10^{12} \\
\text { dynes } / \mathrm{cm}^{2}\end{array}$ \\
\hline Forsterite $\dagger, \mathrm{Mg}_{2} \mathrm{SiO}_{4}$ & 1.249 & 0.714 & 1.286 & 0.811 & -1.8 & -2.6 \\
\hline Olivine, $\uparrow \mathrm{Fo}_{.{ }_{93}} \mathrm{Fa}_{.07}$ & 1.211 & 0.737 . & 1.294 & 0.791 & -1.0 & -2.5 \\
\hline Periclase, $\mathrm{MgO}$ & 0.862 & 0.665 & 1.622 & 1.308 & -0.2 & -1.6 \\
\hline Lime, ${ }^{*} \mathrm{CaO}$ & 1.309 & 0.603 & 1.059 & 0.761 & 0.6 & -3.3 \\
\hline Bromelite, * BeO & 0.538 & 0.0449 & 2.201 & 1.618 & 6.3 & -12.1 \\
\hline Zincite, ${ }^{*} \mathrm{ZnO}$ & 0.613 & -1.138 & 1. 394 & 0.442 & 10.3 & -10.2 \\
\hline Corundum, $\mathrm{Al}_{2} \mathrm{O}_{3}$ & 0.478 & 0.347 & 2.521 & 1.613 & 7.6 & -5.5 \\
\hline Hematite, ${ }^{*} \mathrm{Fe}_{2} \mathrm{O}_{3}$ & 0.591 & 0.151 & 2.066 & 0.910 & 7.7 & -8.1 \\
\hline Spinel, $\mathrm{MgO} .2 \cdot 6 \mathrm{Al}_{2} \mathrm{O}_{3}$ & 0.494 & 0.0762 & 2.020 & 1. 153 & 11.1 & -9.6 \\
\hline Trevorite, $\div \mathrm{NiFe}_{2} \mathrm{O}_{4}$ & 0.610 & -0.0082 & 1.823 & 0.713 & 9.0 & -8.4 \\
\hline Garnet, Al-Py & 0.919 & 0.456 & 1.770 & 0.943 & -1.5 & -4.5 \\
\hline Rutile $\mathrm{TiO}_{2}$ & 0.825 & 0.101 & 2.155 & 1.124 & -3.9 & -9.3 \\
\hline
\end{tabular}

Finite strain parameters $\zeta$ and $\eta$ were computed according to equation 13 .

* Polycrystalline.

$\dagger$ From Kumazawa and Anderson [1969].

† From Liebermann [1969].

$\S$ From Manghnani [1969].

All others from O. L. Anderson et al. [1968].

By fitting the above equations to seismic data, it is possible to evaluate $\lambda_{\nu}, \mu_{0}, \rho_{0}, \zeta$, and $\eta$ for homogeneous regions of the earth's mantle. These material constants can then be compared with ultrasonic measurements and lattice calculations for candidate minerals in an attempt to infer composition. Application of the concepts in this paper to the seismological problem will be treated by the authors in a fortheoming paper. Excellent agreement is found between finite strain theory and the seismic velocities and densities, thereby removing the difficulties experienced by Birch [1939] in his discussion of the lower mantle.

Acknowledgment. This research was supported by NASA Research grant NGL-05-002-069.

\section{REFERENCES}

Anderson; Orson L., E. Schreiber, R. C. Liebermann, and N. Soga, Some elastic constant data on minerals relevant to geophysics, Rev. Geophys., 6, 491, 1968 .

Birch, Francis, The effect of pressure upon the elastic parameters of isotropic solids, according to Murnaghan's theory of finite strain, J. Appl. Phys., 9, 279, 1938.
Birch, Francis, The variation of seismic velocities within a simplified earth model, in accordance with the theory of finite strain, Bull. Seismol. Soc. Amer., 29, 463, 1939.

Bireh, Francis, Elasticity and constitution of the earth's interior, J. Geophys. Res., 57, 227, 1952.

Birch, Francis, Density and composition of the upper mantle: First approximation as an olivine layer, in The Earth's Crust and Upper Mantle, Geophys. Monogr. 13, edited by Pembroke J. Hart, p. 18, Ameriean Geophysical Union, Washington, D. C., 1969.

Hughes, D. S., and J. L. Kelly, Second-order elastic deformation of solids, Phys. Rev., 92, $1145,1953$.

Kumazawa, M., and O. L. Anderson, Elastic moduli, pressure derivatives, and temperature derivatives of single-crystal olivine and singlecrystal forsterite, J. Geophys. Res., 74, 5961, 1969.

Liebermann, R. C., Effect of iron content upon the elastic properties of oxides and some applications to geophysics, Ph.D. thesis, Columbia University, New York, 1969.

Manghnani, M., Elastic constants of single-crystal rutile under pressures to 7.5 kilobars, J. Geophys. Res., 7/4, 4317, 1969.

(Received March 2, 1970; revised May 8, 1970.) 